\title{
Knudsen torque: A rotational mechanism driven by thermal force
}

\author{
Qi Li, Tengfei Liang, and Wenjing Ye* \\ Department of Mechanical and Aerospace Engineering, The Hong Kong University of Science and Technology, \\ Clearwater Bay, Kowloon, Hong Kong
}

(Received 29 April 2014; revised manuscript received 25 August 2014; published 16 September 2014)

\begin{abstract}
Thermally induced mechanical loading has been shown to have significant effects on micro- and nano-objects immersed in a gas with a nonuniform temperature field. While the majority of existing studies and related applications focus on forces, we investigate the torque, and thus the rotational motion, produced by such a mechanism. Our study has found that a torque can be induced if the configuration of the system is asymmetric. In addition, both the magnitude and the direction of the torque depend highly on the system configuration, indicating the possibility of manipulating the rotational motion via geometrical design. Based on this feature, two types of rotational micromotor that are of practical importance, namely pendulum motor and unidirectional motor, are designed. The magnitude of the torque at $\mathrm{Kn}=0.5$ can reach to around $2 \mathrm{nN} \times \mu \mathrm{m}$ for a rectangular microbeam with a length of $100 \mu \mathrm{m}$.
\end{abstract}

DOI: 10.1103/PhysRevE.90.033009

PACS number(s): 47.61.Fg, 47.45.Ab, 47.61.Cb, 05.60.Cd

\section{INTRODUCTION}

Mechanical force induced by thermally nonequilibrium rarefied gas has attracted a significant amount of interest over a long period of time, due to its intriguing mechanism and broad range of applications, particularly in actuation. The wellknown phenomena, radiometric force and thermophoresis, have been well studied and applied in a variety of applications [1-10]. More recently, benefitting from the advanced microfabrication techniques, a relatively new type of thermal force, entitled as Knudsen force, acting on uniformly heated micro- or nanostructures, was discovered and experimentally measured in several microdevices [11-13]. Such a force is significant enough to create an impact on the performance of microdevices, and therefore has provoked a series of numerical and theoretical studies on its fundamental mechanism [14-19]. Generally speaking, Knudsen force is created by gas motion induced by the nonuniform gas temperature. Both the magnitude and the direction of the force depend on the Knudsen number, Kn, a parameter used to measure the rarefaction level of gas, and the maximum force is attained in the transition regime. More intriguingly, the direction of the Knudsen force can also be influenced by the configuration of the system. An asymptotic analysis of the linearized Boltzmann equation reveals in the near continuum regime, thermal stress slip flow is the dominant factor that contributes to the Knudsen force acting on an object with isothermal surfaces, and this flow is determined by the normal temperature gradient around the boundary of the object, which is highly sensitive to the shape of the object [11]. This study points out the fact that the direction of the Knudsen force can be manipulated by designing system configuration, indicating the potential applications of Knudsen force in micro- and nano-object manipulation and separation.

The shape-dependent orientation and magnitude of the Knudsen force also imply that for cases with asymmetric configurations, either due to the asymmetric shape of the object or the asymmetric location of the object with respect to the

*mewye@ust.hk enclosure, a nonzero net torque, denoted as Knudsen torque, possibly exists and acts on the object, in addition to the net force. The presence of such a torque would certainly impact on the performance of microdevices. In the meantime, it could also be utilized as a new type of rotational engine to create rotational motion of micro- and nano-objects, which is often difficult to realize in micro- and nanosystems. Therefore, in this work, a theoretical study based on the asymptotic theory of Boltzmann equation is conducted to investigate the possible Knudsen torque acting on microbeams with different crosssections. Of particular interest are its fundamental mechanism and types of rotational motion it may lead to.

\section{ASYMPTOTIC ANALYSIS}

A two-dimension problem of a uniformly heated microbeam placed inside a cold chamber is considered, and the schematic of the problem domain is illustrated in Fig. 1. The height, the width, and the radius of the corners of the beam and the chamber are denoted by $t L_{0}, l L_{0}, r L_{0}$ and $L_{2} L_{0}$, $L_{1} L_{0}, R L_{0}$, respectively, with $L_{0}$ being the reference length. The centers of the beam and chamber are indicated by $O_{b}$ and $O_{c}$, where $O_{c}=(0,0)$ is set to be the origin of the system. The orientation of the microbeam is indicated by angle $\alpha$ measured clockwise from the horizontal axis. The gap between the horizontally oriented beam and the bottom side of the chamber is denoted by $g L_{0}$. Both the beam and the chamber are isothermal but of different temperatures.

The near-continuum gas behavior inside the chamber is studied using the asymptotic theory based on the linearized Boltzmann equation and fully diffused gas-wall boundary condition [20-22]. The advantage of this approach is that equations of macroscopic physical quantities, such as the temperature, velocity, and pressure field, can be explicitly formulated and are readily used to investigate the underlying fundamental mechanism. Following the same approach employed in our previous work [19], the velocity distribution function and macroscopic variables of gas are expanded as power series of $k=\frac{\sqrt{\pi}}{2} \mathrm{Kn}$. Using the perturbation technique, a set of governing equations and the corresponding boundary 


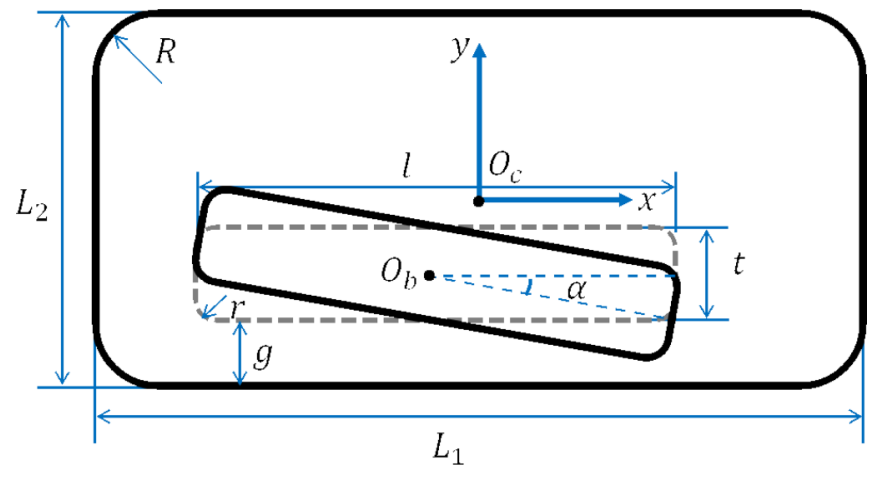

FIG. 1. (Color online) Schematic illustration of a heated microbeam placed inside a cold chamber.

conditions for the macroscopic quantities of various orders can be derived and are summarized in Table I, in which dimensionless variables $P_{i j}, u_{i}$, and $\tau$ denote the nonequilibrium parts of the pressure, velocity, and temperature, respectively. The corresponding macroscopic variables are expressed as: $P_{0}\left(\delta_{i j}+P_{i j}\right),\left(2 R T_{0}\right)^{\frac{1}{2}} u_{i}$, and $T_{0}(1+\tau)$ with $P_{0}$ and $T_{0}$ being the reference values at equilibrium and $R$ being the specific gas constant; the subscript $G$ denotes the Grad-Hilbert solution of gas behavior, and $m$ is the order of macroscopic quantities in terms of Knudsen number, that is, $O\left(k^{m}\right)$; the subscript $w$ indicates the quantities associated with the wall surface; $x_{i}$ is the nondimensional spatial variable; $\mathbf{n}$ is the outward normal vector of the solid boundary and $\mathbf{t}$ is the tangential vector that rotates counterclockwise $90^{\circ}$ from $\mathbf{n}$; and $\gamma_{1}, a_{4}$ are constant coefficients determined by the molecular model. In this work, the hard sphere model and diffuse gas-surface interaction model are employed [23].

The solutions of this set of equations only predict the gas behavior outside the Knudsen layer, that is, a thin gas layer adjacent to the wall boundary with its thickness being on the order of the mean free path of gas. Within the Knudsen layer, a correction is needed in order to obtain the accurate solution. Nevertheless, the moment conservation requires the relations $\frac{\partial P_{i j}}{\partial x_{j}}=0$ and $\frac{\partial P_{i j G}}{\partial x_{j}}=0$ to be satisfied, and hence the force acting on the microbeam immersed in the gas can be obtained simply by integrating stresses over any closed path, $c$, that encloses the microbeam. In addition, the higher-order derivatives of flow velocity and the integration of thermal stress over a closed curve vanish, resulting in only two nonzero terms, $P_{G 3}$ and $S_{i j G 2}$, which contribute to the force and torque as shown in Eqs. (1) and (2):

$$
\begin{aligned}
F_{i} & =-k^{3}\left(\oint_{c}\left(P_{G 3} \delta_{i j}+\gamma_{1} S_{i j G 2}\right) n_{j} d s\right) P_{0} L_{0}, \\
T_{i} & =-k^{3}\left(\oint_{c} \varepsilon_{i l k} x_{l}\left(P_{G 3} \delta_{k j}+\gamma_{1} S_{k j G 2}\right) n_{j} d s\right) P_{0} L_{0}^{2},
\end{aligned}
$$

where $S_{i j G 2}=-\left(\frac{\partial u_{i G 2}}{\partial x_{j}}+\frac{\partial u_{j G 2}}{\partial x_{i}}\right)$ and $\mathbf{n}$ is the outward normal vector of $c$. As indicated in Eqs. (T1)-(T3), both terms result from the bulk flow velocity $u_{i G 2}$, which is in turn caused by the nonuniform normal temperature gradient along the surface [24].

Equations (T1)-(T3) are solved numerically using the boundary element method to obtain gas temperature, flow velocity, and pressure fields. The net force and torque are then calculated by integrating relevant stresses along the closed path $c$ based on Eqs. (1) and (2). The analysis and numerical simulation are validated using a case with two noncoaxial circular cylinders. Knudsen force is calculated and compared with the analytical solution [19], and an agreement within $0.01 \%$ is obtained.

\section{RESULTS AND DISCUSSION}

\section{A. Knudsen torque}

To investigate the Knudsen torque, a system with the following parameters is considered: $t=2, l=10, r=0.4$, $L_{2}=8, L_{1}=20, R=1.0, O_{b}=(0,-1.0)$ and temperature $\tau_{\text {beam }}=0, \tau_{\text {chamber }}=\Delta \tau=-0.1$. The microbeam is oriented at an angle of $\alpha=10^{\circ}$ in order to create an asymmetric configuration. The global Knudsen number is defined based on the gap $g$. Calculations indicate that for such a system, indeed there is a nonzero Knudsen torque acting on the microbeam. The magnitude of the torque is $T_{z}=0.01614 k^{3}$ and its direction is counterclockwise with the rotation center being at the center of the microbeam. Such a torque tends to make the beam return back to its horizontal orientation, that is, $\alpha=0^{\circ}$. Meanwhile, the $x$ and $y$ components of the Knudsen force are found to be negative.

According to the asymptotic analysis, thermal stress slip flow is the driven mechanism for the formation of the Knudsen force acting on the uniformly heated microbeam inside the cold chamber. The net torque is determined by the magnitude and the moment arm of the force, which is distributed along the integration path. To obtain a clear picture of Knudsen torque, the temperature contour, flow structure, and pressure variation

\begin{tabular}{|c|c|c|c|}
\hline & Governing equations & Boundary conditions & \\
\hline Temperature field & $\frac{\partial^{2} \tau_{G 0}}{\partial x_{i}^{2}}=0$ & Beam: $\tau_{\text {beam }}=0$ & $(\mathrm{~T} 1)$ \\
\hline$\left[O\left(k^{0}\right)\right]$ & & Chamber: $\tau_{\text {chamber }}=\Delta \tau$. & \\
\hline $\begin{array}{l}\text { Velocity field } \\
{\left[O\left(k^{2}\right)\right]}\end{array}$ & $\frac{\partial u_{i G 2}}{\partial x_{i}}=0$ & $\begin{array}{c}\left(u_{i G 2}-u_{w i 2}\right) t_{i}=-a_{4} \frac{\partial^{2} \tau_{G 0}}{\partial x_{i} \partial x_{j}} n_{j} t_{i} \\
u_{i G 2} n_{i}=0 .\end{array}$ & $(\mathrm{T} 2)$ \\
\hline $\begin{array}{l}\text { Pressure field } \\
{\left[O\left(k^{3}\right)\right]}\end{array}$ & $\frac{\partial P_{G 3}}{\partial x_{i}}=\gamma_{1} \frac{\partial^{2} u_{i G 2}}{\partial x_{j}^{2}}$ & At a reference point in gas domain: $P_{G 3}=0$ & (T3) \\
\hline
\end{tabular}

TABLE I. Governing equations and boundary conditions for the heated microbeam problem. 


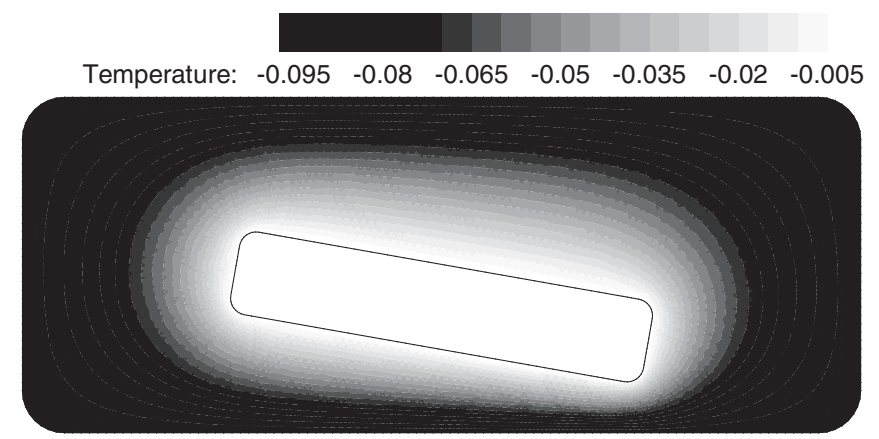

(a)

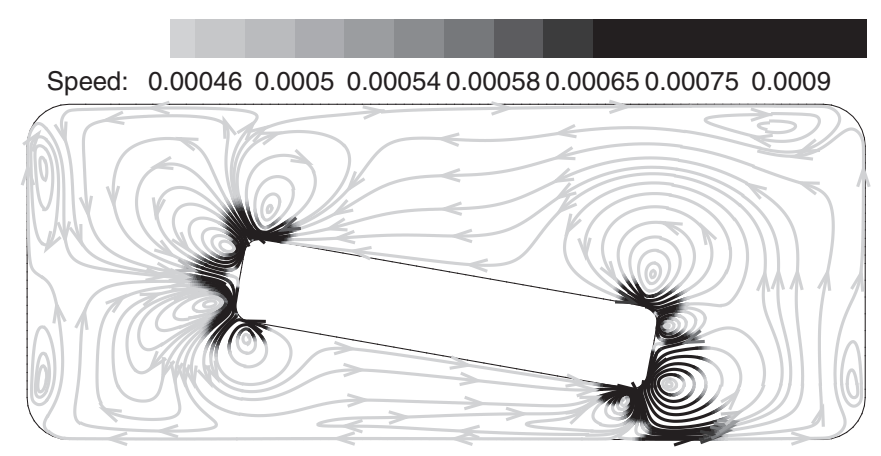

(b)

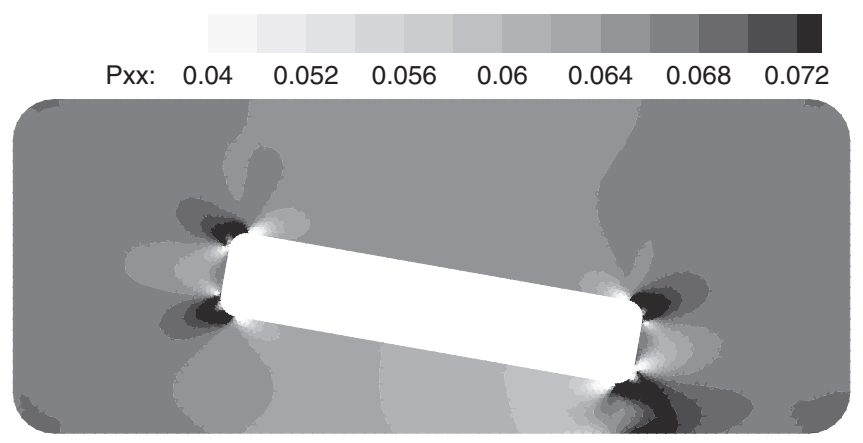

(c)
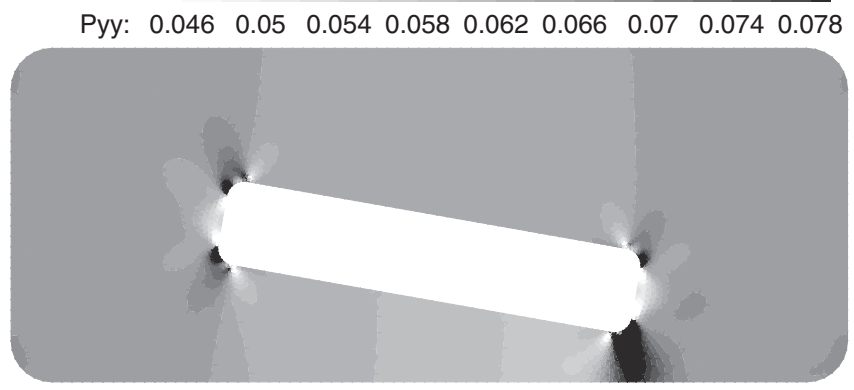

(d)

FIG. 2. Temperature contour, flow structure, and pressure variation of gas surrounding the heated microbeam; (a) temperature distribution; (b) flow structure; (c) pressure $P_{x x}$; (d) pressure $P_{y y}$.

of gas surrounding the heated microbeam are examined and plotted in Fig. 2. It is evident from the plot that the temperature gradient varies rapidly around the corners [Fig. 2(a)], and due to the rarefaction effect, gas flows are induced and vortexes are formed in the vicinity of corners [Fig. 2(b)]. In particular, around the low right corner of the beam, the change in the temperature gradient is mostly significant. As such, more gas molecules are pumped into that corner from the two sides and a higher-pressure region is formed, as indicated clearly in the pressure plots [Figs. 2(c) and 2(d)]. The normal pressure component $P_{x x}$ around the low right corner leads to a clockwise torque, while $P_{y y}$ in the same region yields a counterclockwise torque. Although the magnitudes of $P_{x x}$ and $P_{y y}$ are comparable, the moment arm of $P_{y y}$ is much longer than that of $P_{x x}$. Hence, the net torque due to the pressure is counterclockwise. Meanwhile, the shear stress $P_{x y}$ is found to be one order smaller compared with normal stresses. As a combined effect, the total net torque is dominated by the normal stresses and is counterclockwise. It should be pointed out that the net vertical Knudsen force $F_{y}$ is influenced mostly by the large low-pressure region underneath the beam [Fig. 2(d)], which leads to a downward direction. However, this low-pressure region contributes very little to the torque due to the rather short moment arm.

\section{B. Two types of rotational motion}

As discussed is Sec. III A, the nonzero Knudsen torque comes from the asymmetrically distributed Knudsen force. The Knudsen force, on the other hand, is highly influenced by the shape of the object and the configuration of the system [11]. It is thus expected that a similar dependence exists for Knudsen torque. Indeed, further investigation reveals that it is possible to control the rotational motion of the microbeam and object by designing the configuration of the system. Two types of motion, namely pendulum motion and unidirectional rotation, are considered in this work.

To create a pendulum motion, microbeams with rectangular cross-sections and parameters of $t=2, l=4, r=0.4, L_{2}=8$, $L_{1}=10, R=1.0$ are considered. The center of the beam is fixed at $O_{b}=(0,-1.0)$, and its orientation is controlled by setting angle $\alpha$ at various values. Knudsen forces and torques at different orientations are calculated and shown in Fig. 3, where torques of counterclockwise directions are indicated by positive values. Apart from the horizontal and vertical positions, the microbeam at any other orientation experiences a nonzero torque of which the magnitude changes with its orientation (Fig. 3). In addition, the Knudsen torque reverses its direction from counterclockwise to clockwise when $\alpha>90^{\circ}$ due to the symmetric configurations about the $y$ axis. Hence, the effect of the Knudsen torque is to bring the beam back to its horizontal position from any other nonvertical position and pendulum motion is created. If the beam is orientated vertically, it would remain vertical since the net Knudsen torque at vertical position is zero. However, any small perturbation would cause the beam to go back to its horizontal position, indicating that the vertical position is unstable while the horizontal position is stable.

To have more stable positions would allow flexibility in the design of pendulum motors and thus broaden the 


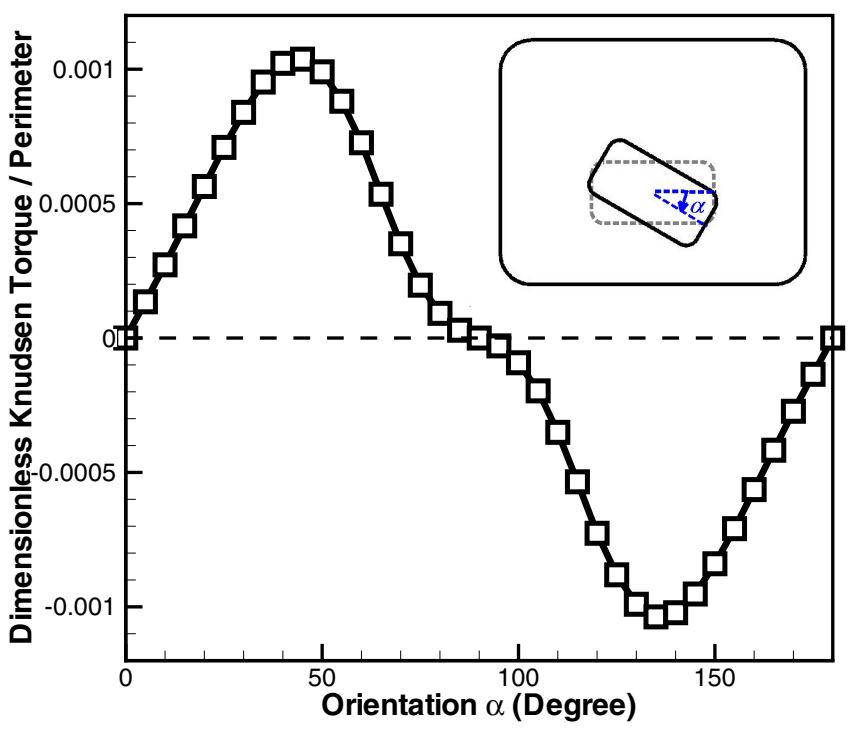

FIG. 3. (Color online) The Knudsen torque acting on a rectangular microbeam as a function of the orientation of the beam located at $O_{b}=(0,-1.0)$.

application scope. This can be realized by changing the system configuration as demonstrated in Fig. 4. When the microbeam is fixed at $O_{b}=(-2.0,-1.0)$, multiple stable positions are found. In particular, two stable positions are found to be around $\alpha=5^{\circ}$ and $\alpha=85^{\circ}$. The other two equilibrium positions corresponding to $\alpha=53^{\circ}$ and $\alpha=117^{\circ}$ are unstable. By pushing the beam further away from the center, more stable positions can be found.

To realize the pendulum motion, one must also consider the gas damping force acting on the rotating microbeam. In the near continuum regime, the dissipation is expected to be high, which may result in no oscillation. Thus, it is necessary

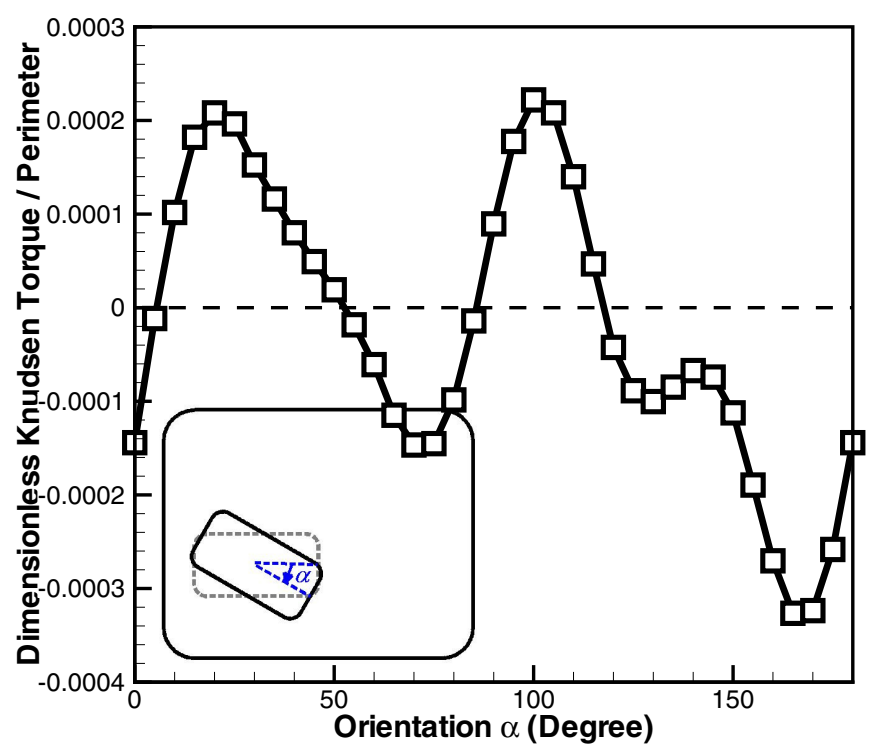

FIG. 4. (Color online) The Knudsen torque acting on the microbeam as a function of the orientation of the beam located at $O_{b}=(-2.0,-1.0)$.

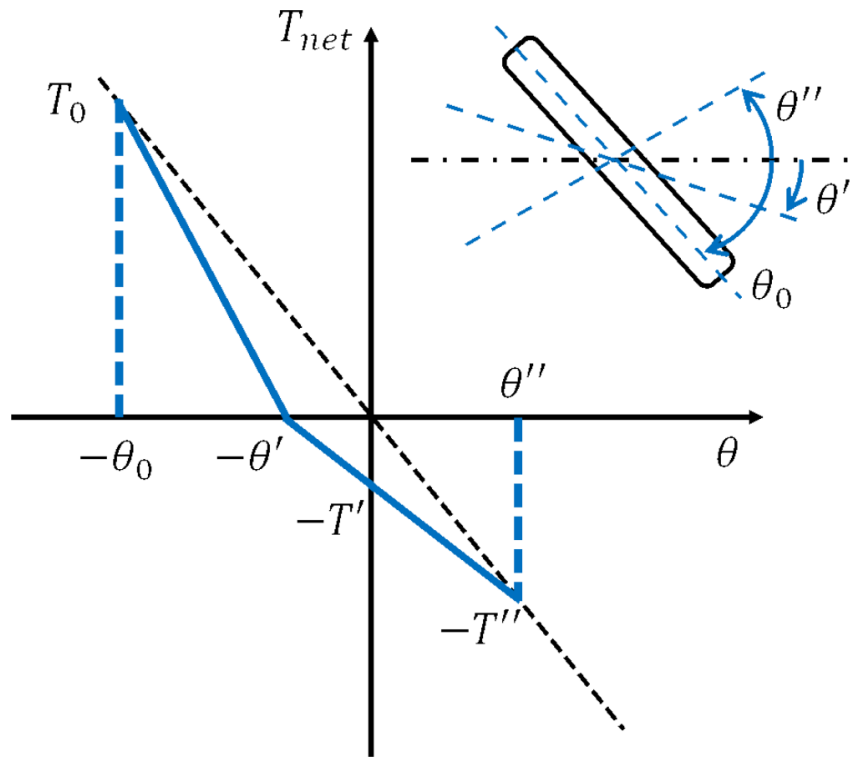

FIG. 5. (Color online) The relationship between the net torque indicated by the solid line and the rotating angle $\theta$. The dashed line represents the Knudsen torque, that is, the thermally induced torque.

to estimate the dumping rate to evaluate the feasibility of the pendulum motion. Consider the case when the beam is initially oriented $\theta_{0}$ clockwise from the stable horizontal position as shown in Fig. 5 and is at rest. The thermally induced torque rotates the beam counterclockwise. Assume the beam passes through the horizontal position and reaches to zero velocity at a position with an angle of $\theta^{\prime \prime}$. The dumping rate can be estimated by calculating the ratio of $\eta=\frac{\theta_{0}-\theta^{\prime \prime}}{\theta_{0}}$, which is denoted as the damping factor.

As shown in Fig. 5, the Knudsen torque, which was calculated previously (Fig. 3), is approximatively linear with respect to angle $\theta$ in the range of $-30^{\circ} \leqslant \theta \leqslant 30^{\circ}$. The viscous drag torque, as listed in Table II, is also near linear with respect to the angular velocity at $\theta=0$. Hence, it is reasonable to assume that the net torque acting on the microbeam shown by the solid line in Fig. 5 is linear piecewise. It reaches to zero at $\theta^{\prime}$, at which the viscous drag torque reaches to its maximum and balances out the Knudsen torque. The viscous drag torque acting on the beam at $\theta=0$, is calculated and fitted with a linear relation with the angular velocity $T^{\prime}=a \dot{\theta}$ (Table II). By considering work-energy conversion and the linear relationship between the net torque and the angle $\theta$, a set of equations can be formulated to roughly estimate the

TABLE II. Viscous drag torque acting on the micrbeam at the horizontal position $\theta=0$ with different angular velocities.

\begin{tabular}{lc}
\hline \hline Angular velocity & Viscous drag torque \\
$\dot{\theta}\left(k^{2} \sqrt{2 R T} / L_{0}\right)$ & $T^{\prime}\left(k^{3} P_{0} L_{0}^{2}\right)$ \\
\hline $10^{-4}$ & $8.186 \times 10^{-3}$ \\
$10^{-5}$ & $8.193 \times 10^{-4}$ \\
$10^{-6}$ & $8.358 \times 10^{-5}$ \\
\hline
\end{tabular}




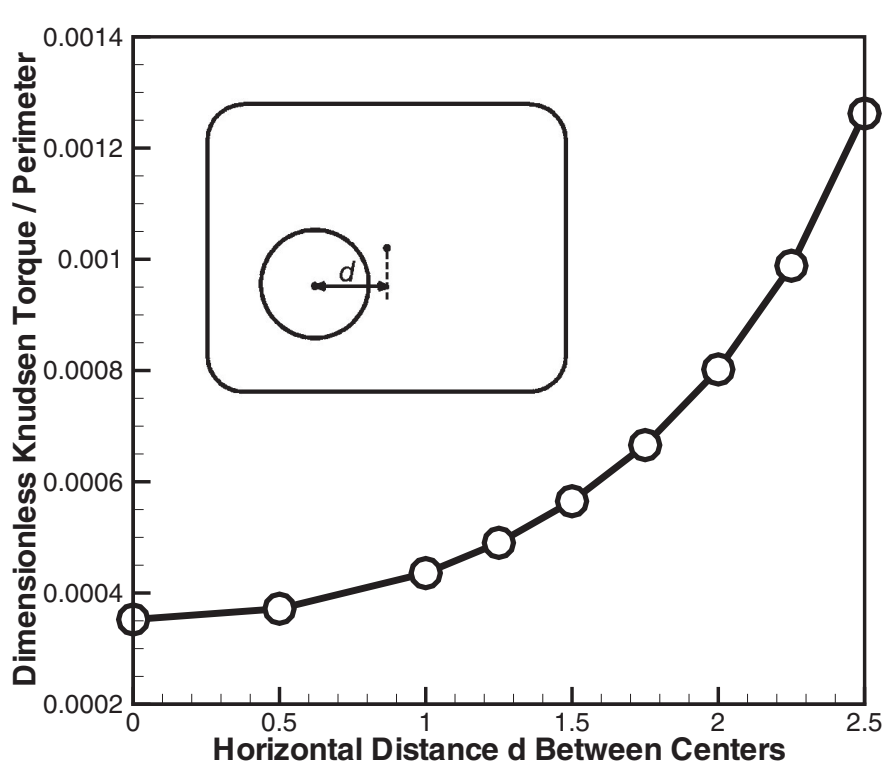

(a)

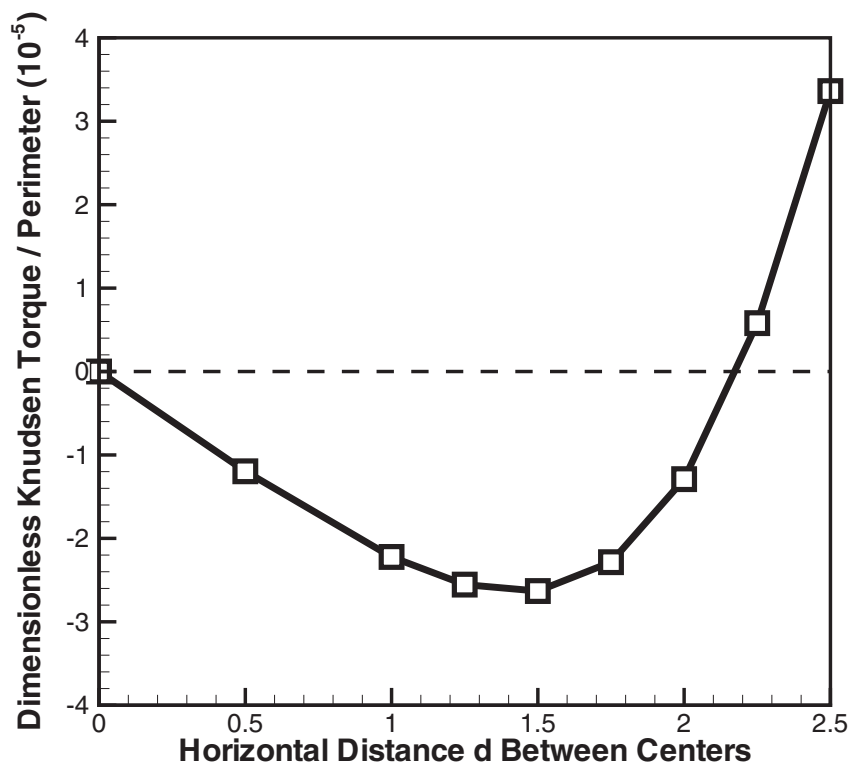

(b)

FIG. 6. The Knudsen force (a) and torque (b) acting on the cylinder as functions of the horizontal distance $d$ between the center of the chamber and the center of the cylinder.

damping factor as listed as follows.

$$
\begin{aligned}
\text { Knudsen torque linearly changed with } \theta & : T^{\prime \prime}=T_{0} \frac{\theta^{\prime \prime}}{\theta_{0}}, \\
\text { Net torque linearly changed with } \theta: & \frac{\theta^{\prime}}{\theta^{\prime \prime}}=\frac{T^{\prime}}{T^{\prime \prime}-T^{\prime}}, \\
\text { Fitting from calculation at } \theta=0: & T^{\prime}=a \dot{\theta},
\end{aligned}
$$

Work-energy theorem from $\theta_{0}$ to $\theta=0$, and net torque linearly changed with $\theta: \frac{1}{2}\left(\theta_{0}-\theta^{\prime}\right) T_{0}-\frac{1}{2} \theta^{\prime} T^{\prime}=\frac{1}{2} J \dot{\theta}^{2}$,

Work-energy theorem from $\theta_{0}$ to $\theta^{\prime \prime}, \quad$ and net torque linearly changed with $\theta: \frac{1}{2}\left(\theta_{0}-\theta^{\prime}\right) T_{0}=\frac{1}{2} T^{\prime \prime}\left(\theta^{\prime}+\theta^{\prime \prime}\right)$,

where $J$ is the moment of inertia of the microbeam. The dumping factor is solved as,

$$
\eta=\frac{\theta_{0}-\theta^{\prime \prime}}{\theta_{0}}=\frac{1}{2 J T_{0}}\left(a^{2} \theta_{0}+a \sqrt{a^{2} \theta_{0}^{2}+4 J T_{0} \theta_{0}}\right) .
$$

Let $\theta_{0}=-30^{\circ}, T_{0}=0.0096 k^{3} P_{0} L_{0}^{2}$, temperature $T=$ $300 \mathrm{~K}$, and the reference pressure is $10^{2} \mathrm{~Pa}$. Assume the beam is made of silicon and its density is $2330 \mathrm{~kg} / \mathrm{m}^{3}$. The fitting parameter $a=82 k P_{0} L_{0}^{3} / \sqrt{2 R T}$. The calculated dumping factor is about $\eta=84.5 \%$ at $\mathrm{Kn}=0.02$, and $\eta=65.3 \%$ at $\mathrm{Kn}=0.03$.

While the dumping rate is considerably large in the near continuum regime, it can be greatly reduced in the transition regime, at which the Knudsen torque will increase but the drag torque will be reduced. Thus, the pendulum motion is feasible to be created especially in the highly rarefied system.

The main reason that the pendulum motion can be realized in the microbeam system is that when the rectangular beam rotates the relative position of the beam with respect to the chamber changes. As such, both the direction and magnitude of the Knudsen torque change due to the dependence of Knudsen torque on the system configuration, and a pendulum motion is created. To design for continuous unidirectional rotations, the system must be such that its configuration does not change as the rotor rotates. A suitable candidate for the rotor would be a cylinder. In this work, a cylinder with a radius of $r=1.5$ inside a cold chamber is considered. The chamber's width, length, and radius of its corners are set to be $L_{2}=8, L_{1}=10$, $R=1.0$, respectively.

For a cylinder, a nonzero Knudsen torque is only possible if the cylinder is off the center of the chamber. Various locations of the cylinder along the line $y=-1.0$ are considered. The corresponding Knudsen forces and torques acting on the cylinder are calculated and results for cases with the cylinder located in the range between $(0,-1.0)$ to $(-2.5,-1.0)$ are plotted in Fig. 6. At each location, the Knudsen torque is a constant vector independent of the rotation of the cylinder. Hence, the cylinder would undergo unidirectional rotation and a continuous unidirectional rotor is created. It is also interesting to note that while the Knudsen force always points upward, regardless of the location of the cylinder, Knudsen torque switches its direction when the cylinder gets close to the chamber, at around $(-2.17,-1.0)$. To probe the mechanism of such a switch, the flow structures of gas in two selected cases are examined in details and are shown in Fig. 7. In one case, the cylinder is fixed at $O_{b}=(-1.5,-1.0)$, whereas 


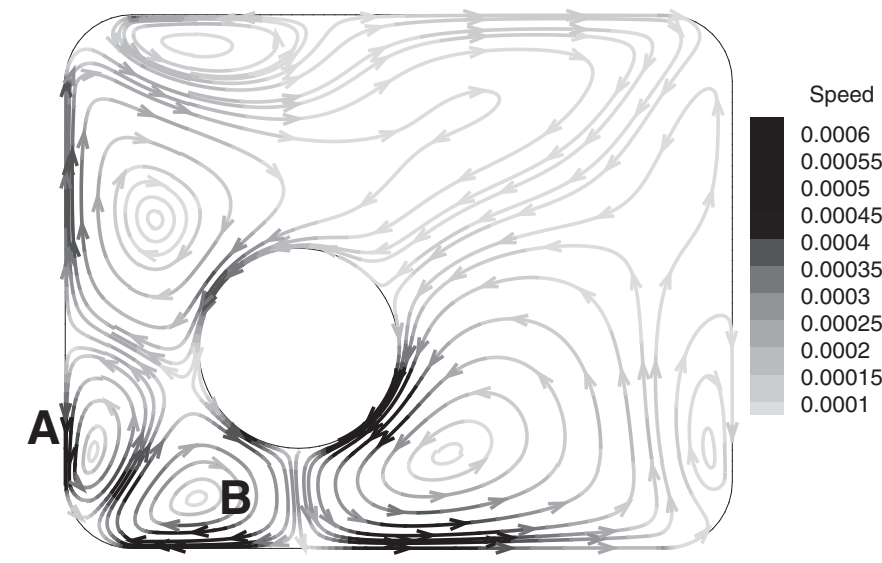

(a)

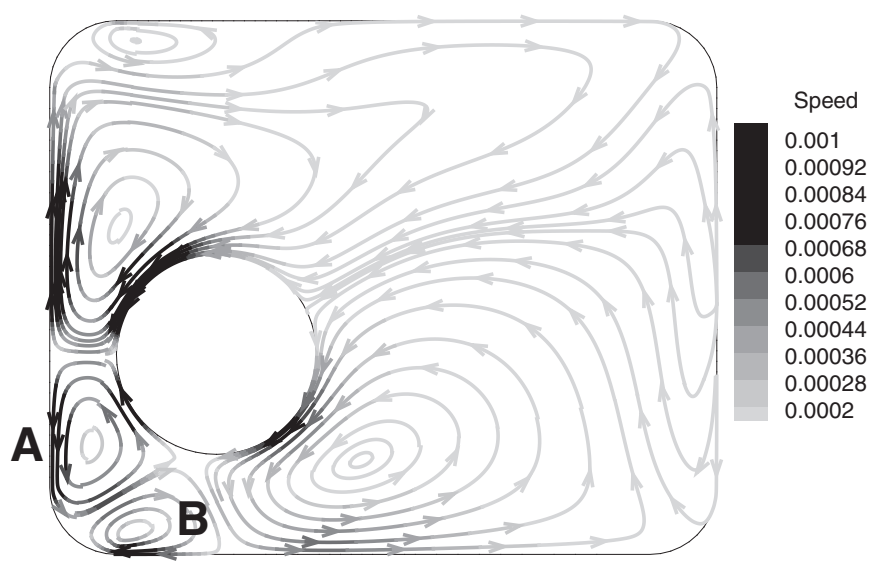

(b)

FIG. 7. The flow structure of gas surrounding the heated cylinder: (a) $O_{b}=(-1.5,-1.0)$; (b) $O_{b}=(-2.5,-1.0)$.

in the other case, the cylinder is fixed at $O_{b}=(-2.5,-1.0)$. The rotational directions in the two cases are opposite. The origin of the Knudsen force, and thus the Knudsen torque, is the thermally induced gas flow, which redistributes gas molecules and creates nonuniform stress fields [8]. In the example considered, thermal stress slip flow, induced by the nonuniform normal temperature gradient along the cylinder, is the dominant flow [11]. The unbalanced momentum resulting from the collisions between gas molecules and the cylinder drives gas molecules to form a bulk flow near the boundary. In the meantime, it also exerts a force on the cylinder which is opposite to the direction of the bulk flow near the surface. Hence, the torque produced by this force has the same direction as that of the vortex formed by the bulk flow near the cylinder. As shown in Fig. 7, the flow structures in the two cases are quite similar. Both have four vortexes near the cylinder and the directions of the two large vortexes are the same in the two cases. The only difference lies in the two relatively small vortexes near the left corner of the chamber. In Fig. 7(a), vortex $\mathrm{B}$ is attached to the cylinder while vortex $\mathrm{A}$ is detached from the cylinder. Therefore, the torque produced has the direction of vortex B. However in Fig. 7(b), it is vortex A that is attached to the cylinder. The opposite directions of vortexes A and B lead to the change in the direction of the net torque from clockwise in Fig. 7(a) to counterclockwise in case Fig. 7(b).

For practical applications, it is important to estimate the rotational speed that is achievable by such a mechanism. As the cylinder rotates, a resisting torque develops due to the viscous drag force. Hence, the steady-state speed can be calculated by equaling the two torques. A rough estimation has found that for the case with $O_{b}=(-1.5,-1.0)$, the angular speed of the beam is around $\omega=5.6 \times 10^{-6} k^{2} \sqrt{2 R T} / L_{0}$, that is, for the length scale $L_{0}=1 \mu \mathrm{m}, T=300 \mathrm{~K}$, and $k=5 \times 10^{-3}$, a rotational frequency of $0.56 \mathrm{rpm}$ can be achieved. This speed can be increased by either reducing the characteristic length of the object, or by increasing the Knudsen number, $k$.

\section{Knudsen torque in the transition regime}

In the near continuum regime, the gas surrounding the object is only slightly rarefied and deviated from the equi- librium, resulting in a small Knudsen force and thus a small Knudsen torque. It has been numerically and experimentally demonstrated that the magnitude of Knudsen force can have several orders of magnitude increase in the transition regime $[11,12,16]$. Such a fact implies that the Knudsen torque can also be greatly increased in the transition regime. To demonstrate, a system with a rectangular microbeam is considered. The dimensions of the system are such that $t=2$, $l=4, L_{2}=8, L_{1}=10, O_{b}=(-2.0,-1.0)$, with reference length $L_{0}=1 \mu \mathrm{m}$. The temperatures on the microbeam and chamber are set to be 500 and $300 \mathrm{~K}$, respectively.

For highly rarefied systems, the asymptotic theory is no longer valid and a direct simulation Monte Carlo (DSMC) study with fully diffused gas-wall boundary condition is conducted to calculate the Knudsen torque acting on the microbeam. The simulation parameters in DSMC, such as cell size and time step, are chosen according to Ref. [16]. The case at which the maximum Knudsen force was numerically demonstrated [16] and experimentally observed [11,12] is simulated and the corresponding Knudsen number is 0.5 . The calculated Knudsen forces are $F_{x}=-0.37 \mathrm{nN} / \mu \mathrm{m}$ and $F_{y}=$ $-0.10 \mathrm{nN} / \mu \mathrm{m}$, respectively, and the Knudsen torque is $T_{z}=$ $-0.016 \mathrm{nN} \times \mu \mathrm{m} / \mu \mathrm{m}$. These results were validated using another DSMC code provided by G. A. Bird. The agreement among the forces is within $10 \%$, while the agreement between the torques is within $23 \%$. Compared with the results from the same system but with rounded corners at $\mathrm{Kn}=0.005$, the magnitude of Knudsen torque increases about five orders of magnitude, $1.13 \times 10^{5}$, indicating its feasibility in practical applications. It should be pointed out that for rectangular beam with sharp corners, edge-induced thermal flows play an important role in the formation of Knudsen force and torque. The induced torque is, in fact, in the opposite direction of that acting on a beam with rounded corners at small Knudsen numbers.

\section{CONCLUSION}

Using the asymptotic analysis for Boltzmann equation in the near continuum regime, the Knudsen torque acting on an asymmetrically oriented uniformly heated microbeam in a cold 
enclosure is investigated. Due to the shape effect on the force orientation and also its distribution along the surface of the microbeam, the torque is proven to be present and is tunable via system configuration. Two types of rotational motion are designed: the pendulum motion of a rectangular beam with multiple stable positions and the continuous unidirectional rotation of a cylindrical beam. A notable rotational speed has been demonstrated on the cylinder, which shows its potential to serve as a new type of rotor. Furthermore, DSMC simulations indicate that the magnitude of the Knudsen torque can be increased by five orders of magnitude in the transition regime, making thermally driven rotors more attractive for practical applications in micro- and nanosystems in which gas is often in the transition regime.

\section{ACKNOWLEDGEMENTS}

This work is in part supported by Hong Kong University of Science and Technology under Research Project Competition Grant No. RPC11EG37. We are very grateful to Professor G. B. Bird for providing us a highly efficient DSMC code.
[1] N. Selden et al., J. Fluid Mech. 634, 419 (2009).

[2] N. Selden et al., Phys. Rev. E 79, 041201 (2009).

[3] A. Ketsdever et al., Vacuum 86, 1644 (2012).

[4] J. Tyndall, Proc. R. Inst. 6, 1 (1870).

[5] J. R. Brock, J. Colloid Sci. 17, 768 (1962).

[6] Y. Sone, Phys. Fluids 15, 1418 (1972).

[7] Y. Sone and K. Aoki, Phys. Fluids 20, 571 (1977).

[8] M. M. R. Williams, J. Phys. D: Appl. Phys. 19, 1631 (1986).

[9] S. Takata and Y. Sone, Eur. J. Mech. B/Fluids 14, 487 (1995).

[10] F. Zheng, Adv. Colloid Interface Sci. 97, 255 (2002).

[11] A. Passian et al., Phys. Rev. Lett. 90, 124503 (2003).

[12] A. Passian et al., Ultramicroscopy 97, 401 (2003).

[13] B. Gotsmann and U. Dürig, Appl. Phys. Lett. 87, 194102 (2005).

[14] A. Passian et al., J. Appl. Phys. 92, 6326 (2002).
[15] A. L. Lereu et al., Appl. Phys. Lett. 84, 1013 (2004).

[16] T. Zhu and W. Ye, Phys. Rev. E 82, 036308 (2010).

[17] J. Nabeth, S. Chigullapalli, and A. A. Alexeenko, Phys. Rev. E 83, 066306 (2011).

[18] T. Zhu, W. Ye, and J. Zhang, Phys. Rev. E 84, 056316 (2011).

[19] Q. Li, T. Liang, and W. Ye, Phys. Rev. E 88, 033020 (2013).

[20] Y. Sone, in Rarefied Gas Dynamics, edited by L. Trilling and H. Y. Wachman (Academic Press, New York, 1969), p. 243.

[21] Y. Sone, in Rarefied Gas Dynamics, edited by D. Dini (Editrice Tecnico Scientifica, Pisa, 1971), p. 737.

[22] Y. Sone, Annu. Rev. Fluid Mech. 32, 779 (2000).

[23] S. Takata and M. Hattori, J. Stat. Phys. 147, 1182 (2012).

[24] Y. Sone, and S. Tanaka, in Theoretical and Applied Mechanics, edited by F. P. J. Rimrott, and B. Tabarrok (North-Holland, Amsterdam, 1980), p. 405. 